\title{
Improvement After Thalidomide and Dexamethasone Treatment for Advanced Cardiac Amyloidosis A Case Report
}

\author{
Jin Sung Choi, MD; Eui Nam Hwang, MD; Young-Hoon Kim, MD; Yunjin Jung, MD; \\ Hye-Jeong Kim, MD; Seung-Hyun Nam, MD; Joong-Il Park, MD
}

\begin{abstract}
A case of advanced cardiac amyloidosis was treated with thalidomide. The patient showed early and significant improvement of diastolic cardiac function, as well as symptoms. (Circ J 2007; 71: 1823-1825)
\end{abstract}

Key Words: Amyloidosis; Heart failure; Thalidomide

A myloidosis is a disease characterized by the deposition of insoluble fibrous protein extracellularly in several organs. Cardiac amyloidosis (CA) occurs in more than $50 \%$ of cases with light-chain amyloidosis or hereditary amyloidosis; the prognosis of affected patients is poor, with an average survival time of 1-2 years after diagnosis. When heart failure is present, the survival time is as short as 6 months $!^{1-3}$ The available methods for treating amyloidosis have many associated problems, which is an especial concern for advanced CA presenting as heart failure because of the time needed for remission and the toxicity of the drugs? In the present case, treatment with thalidomide in a patient diagnosed with CA and diastolic heart failure lead to an early and significant improvement of symptoms and cardiac function.

\section{Case Report}

A 62-year-old man was admitted for dyspnea that had worsened over the past 2 months prior to admission. The physical findings were raccoon eyes (Fig 1A), multiple ecchymoses, with highly friable skin, on the face and the upper limbs, bilateral rales at the bottom of the lungs, and pedal edema. Chest radiography showed cardiomegaly and pulmonary congestion (Fig 1B). Electrocardiography showed low voltage at the limb leads and poor $\mathrm{R}$ progression in the precordial leads (Fig 1C). The laboratory evaluations revealed proteinuria $(1,621 \mathrm{mg} /$ day), increased level of $B$ type natriuretic peptide (BNP) $(2,733 \mathrm{pg} / \mathrm{ml})$, aspartate aminotransferase $(137 \mathrm{U} / \mathrm{L})$, and alanine transaminase (63 U/L). There was Bence-Jones proteinuria on urine immunofixation electrophoresis. Polarizing microscopy of abdominal subcutaneous tissue showed amyloid and the typical apple-green birefringence after Congo red staining (Fig 1D). Initial transthoracic echocardiography (TTE) showed severe left ventricular (LV) diastolic dysfunction, myocardial thickening $(22 \mathrm{~mm})$, accompanied by granular

(Received April 8, 2007; revised manuscript received May 30, 2007; accepted July 10, 2007)

Department of Internal Medicine, Seoul Veterans Hospital, Seoul, Korea

Mailing address: Joong-Il Park, MD, Department of Internal Medicine, Seoul Veterans Hospital, 6-2 Dunchon Dong, Gangdong Gu, Seoul, 134-791, Korea. E-mail: drjipark@naver.com sparkling, thickening of mitral leaflets and mild LV systolic dysfunction (Figs 2A,B). Based on these findings the patient was diagnosed with diastolic heart failure as a complication of CA. Because CA was advanced and could not be treated by the existing standard treatment regimen, the patient was started on thalidomide $(200 \mathrm{mg} /$ day $)$ and dexamethasone (stopped after initial 6 months) as a proposed new treatment option. Treatment-related toxicity, such as anorexia, constipation and depression, occurred initially, but gradually subsided. After treatment, the degree of dyspnea improved from New York Heart Association class grade IV to grade II, the ecchymoses disappeared and the edema in the lower limbs decreased. There were positive changes in the electrocardiographics findings, such as improvement of low voltage and disappearance of the poor $\mathrm{R}$ progression, at 3-month follow-up. Light-chain amyloid was not found on protein electrophoresis after the $3^{\text {rd }}$ month of treatment, and the proteinuria disappeared after 5 months of treatment. On the follow-up TTE, the granular sparkling had begun to decrease significantly and the cardiac function showed improvement. In particular, the diastolic function showed remarkable improvement, from an irreversible restrictive pattern (Grade IV) to a pseudonormal pattern (Grade II) (Figs 2C-F, Table 1), consistent with improvement of heart failure symptoms, although there was no significant decrease in the thickness of the cardiac wall or in the level of BNP (Table 1). On the latest follow-up (15 months) at the outpatient clinic, the patient is stable, and laboratory and echocardiographic findings show sustained complete response.

\section{Discussion}

Several methods of treating AL amyloidosis with chemotherapy or organ transplantation have had variable results. High-dose melphalan with autologous stem-cell transplantation has been the mainstay of treatment and complete remission is possible in some patients. However, these treatments are limited for cases of CA with heart failure because of the treatment-related mortality, and in cases of AL amyloidosis invading the heart, there is no effective treatment?,4-6 Therefore, advanced CA has been considered as untreatable and affected patients receive conservative treatment. CA is the leading cause of death in patients with 

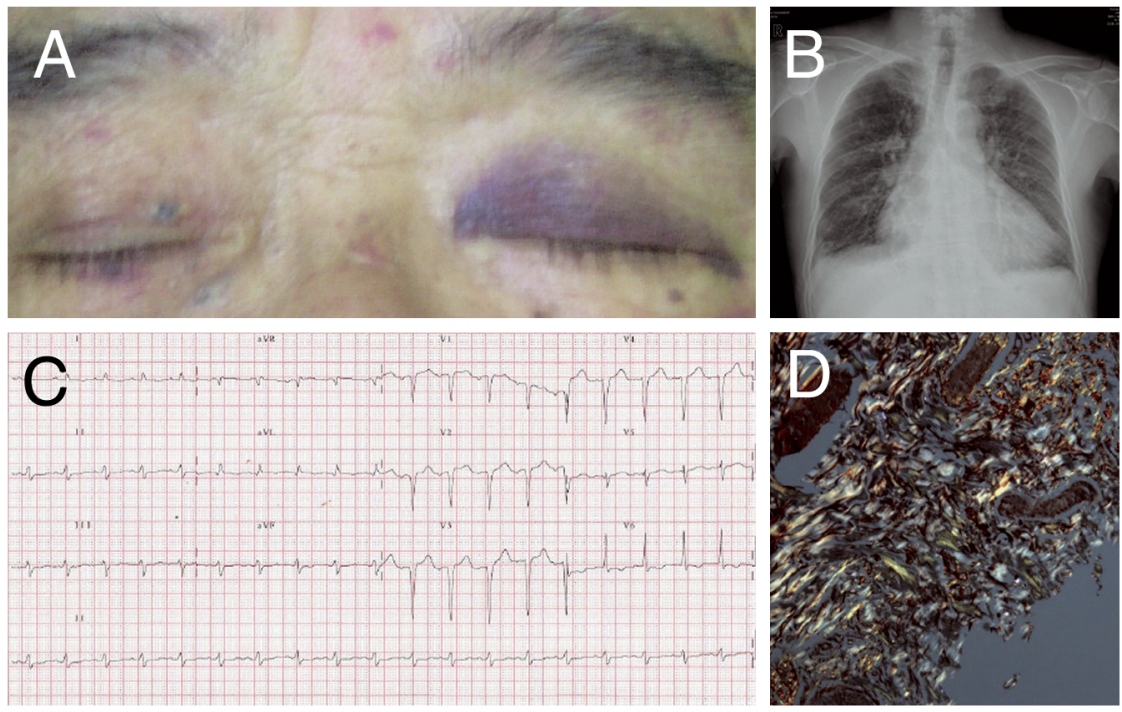

Fig 1. (A) Raccoon eyes. (B) Chest radiography shows cardiomegaly and pulmonary congestion. (C) Electrocardiography shows low voltages in the limb leads and poor $\mathrm{R}$ progression in the precordial leads. (D) Polarizing microscopy of abdominal subcutaneous tissue shows amyloid with polarized light and the typical apple-green birefringence after Congo red staining.
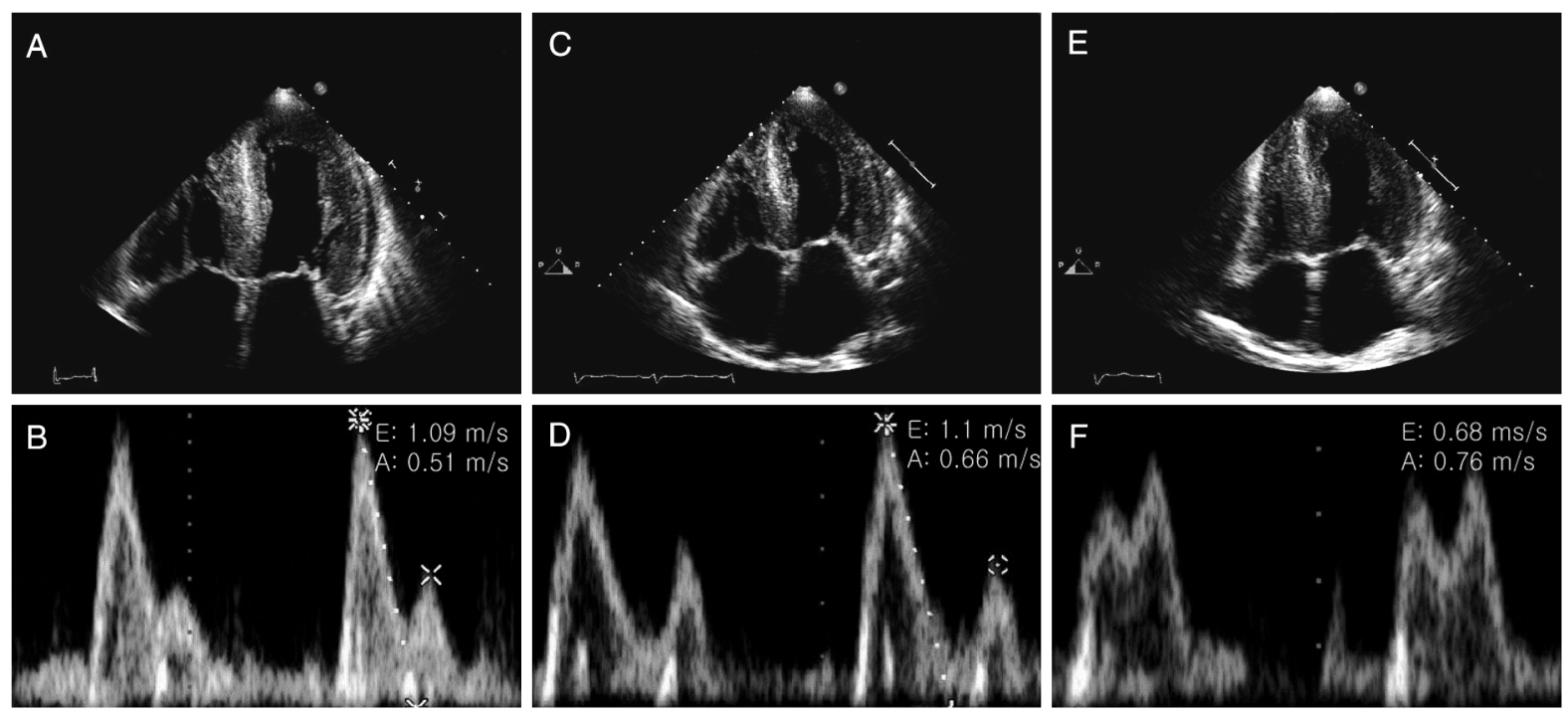

Fig 2. Echocardiographic findings. Initial echocardiography (Panels A,B) shows severe left ventricular diastolic dysfunction and myocardial thickening accompanied by granular sparkling. Follow-up echocardiography at 4 months (Panels C,D) and 11 months (Panels E,F) shows a significant decrease in the granular sparkling, and improvement of the the E/A ratio and deceleration time of the early mitral inflow compared with the initial evaluation. However, the thickness of left ventricular wall has not decreased. E, early diastolic filling velocity; A, filling velocity after atrial contration.

Table 1 Clinical Course and Echocardiographic Parameters

\begin{tabular}{lcccccc}
\hline \hline & Initial & \multicolumn{5}{c}{ Months } \\
\cline { 3 - 6 } & & 3 & 4 & 6 & 8 & 11 \\
\hline Thalidomide $(\mathrm{mg})$ & 200 & 200 & 200 & 200 & 200 & 200 \\
EF $(\%)$ & 43 & 50 & 49 & 53 & 52 & 54 \\
DT (ms) & 150 & 173 & 197 & 165 & 171 & 197 \\
E/A & 2.1 & 2.0 & 1.7 & 1.2 & 1.5 & 0.9 \\
E/E & 40 & 39.6 & - & - & - & 20 \\
IVSd (mm) & 23 & 23 & 23 & - & 22 & 22 \\
LVPWd (mm) & 22 & 22 & 22 & - & 22 & 22 \\
BNP & 2,733 & 3,397 & 3,308 & 3,274 & 2,613 & 2,816 \\
CTR & 0.65 & 0.62 & - & 0.64 & 0.68 & 0.68 \\
\hline
\end{tabular}

$E F$, ejection fraction; $D T$, deceleration time; $E$, early diastolic filling velocity; A, filling velocity after atrial contration; E', early diastolic mitral annulus velocity; IVSd, interventricular septum, diastole; LVPWd, left ventricular posterior wall, diastole (mm); BNP, B-type natriuretic peptide; CTR, cardiothoracic ratio. 
systemic amyloidosis, and the overall prognosis still remains poor, although there are new emerging therapies, including thalidomide, bortezomib, and lenalidomide?

Patients treated with thalidomide have been reported to have improvement of symptoms and organ function, but without improvement of CA4 Interestingly, in the present case, which we treated with thalidomide, we noted a striking improvement of echocardiographic findings and a rapid, sustained complete response of the laboratory parameters, as well as an extension of survival time. These findings suggest that thalidomide could be used as an alternative treatment regimen for cases of CA and heart failure. The possible matrix stabilizing effect and anti-inflammatory effect of thalidomide, which improve the granular sparkling and decrease heart mass, might improve diastolic heart function?

Previous reports proposed that increased BNP levels, LV wall thickness greater than $15 \mathrm{~mm}$ and impaired systolic function are poor prognostic factors?,8 In particular, BNP level has been reported to be more sensitive than conventional echocardiographic parameters in detecting clinical improvement or worsening of CA during follow-up8 However, in the present case, neither the BNP level nor the thickened LV wall showed significant changes and did not reflect the response to treatment at follow-up. Instead, improvement of diastolic function was accompanied by improvement of symptoms.

It is well known that the BNP level increases when the $\mathrm{LV}$ is stressed, and it acts as a protective agent, preventing hypertrophy and fibrosis of cardiomyocytes. Nils et al have reported that the level of $\mathrm{BNP}$ is more related to the preoperative LV mass, rather than being a symptom, and elevated levels are maintained up to 12 months following surgery for aortic stenosis, which is similar to the present case and suggest that either there may not be a correlation between BNP and symptoms ${ }^{10}$ or a longer follow up period is needed to observe a decrease in the BNP level! 10

Therefore, this case illustrates the usefulness of close echocardiographic monitoring of diastolic function for evaluating the treatment response of CA.

\section{References}

1. Kristen AV, Meyer FJ, Perz JB, Schonland SO, Hundemer M, Hegenbart U, et al. Risk stratification in cardiac amyloidosis: Novel approaches. Transplantation 2005; 80: 151-155.

2. Falk RH. Diagnosis and management of the cardiac amyloisodes. Circulation 2005; 112: 2047-2060.

3. Falk RH, Comenzo RL, Skinner M. The systemic amyloidosis. $N$ Engl J Med 1997; 337: 898-909.

4. Palladini G, Perfetti V, Perlini S, Obici L, Lavatelli F, Caccialanza R, et al. The combination of thalidomide and intermediate-dose dexamethasone is an effective but toxic treatment for patients with primary amyloidosis. Blood 2005; 105: 2949-2951.

5. Kyle RA, Gertz MA, Greipp PR, Witzig TE, Lust JA, Lacy MQ, et al. A trial of three regimens for primary amyloidosis: Colchicine alone, melphalan and prednisone, and melphalan, prednisone, and colchicine. N Engl J Med 1997; 336: 1202-1207.

6. Sanchorawala V, Wright DG, Seldin DC, Falk RH, Berk JL, Dember LM, et al. Low-dose continuous oral melphalan for the treatment of primary systemic (AL) amyloidosis. Br J Haematol 2002; 117: 886889.

7. Palladini G, Campana C, Klersy C, Balduini A, Vadacca G, Perfetti $\mathrm{V}$, et al. Serum N-terminal pro-brain natriuretic peptide is a sensitive marker of myocardial dysfunction in AL amyloidosis. Circulation 2003; 107: 2440-2445.

8. Neil M, Simon GR. Cardiac amylodosis. Clin Med 2005; 5: 504-509.

9. Nils ON, Cathrine WK, Trygve H, Øystein AV, John P, Mons L, et al. The effect of aortic valve replacement on plasma B-type natriuretic peptide in patients with severe aortic stenosis: One year follow-up. Eur J Heart Fail 2006; 8: 257-262.

10. Lars G, Thor U, Jan G F, Even H, Torstein G, Kjell B, et al. Effect of thalidomide on cardiac remodeling in chronic heart failure results of a double-blind, placebo-controlled study. Circulation 2005; 112: $3408-3414$. 